\title{
Detección y tipificación del Virus Papiloma Humano en el marco del tamizaje virológico para la detección de lesiones del cuello uterino en Asunción, Paraguay
}

\author{
* María Liz Bobadilla ${ }^{1}$, Verónica Villagra ${ }^{1}$, María Elena Zorrilla ${ }^{1}$, Gladys Olmedo ${ }^{1}$, María Cristina Riveros ${ }^{2}$, \\ Francisco Franco ${ }^{2}$, Gladys Roscher ${ }^{2}$, Hernando Rodríguez ${ }^{3}$, Diana Giménez ${ }^{3}$, Cristina González Bado ${ }^{4}$, \\ Jorge Basiletti ${ }^{5}$, María Alejandra Picconi ${ }^{5}$ \\ ${ }^{1}$ Ministerio de Salud Pública y Bienestar Social (MSPyBS), Laboratorio Central de Salud Pública (LCSP). Asunción, \\ Paraguay \\ ${ }^{2}$ Ministerio de Salud Pública y Bienestar Social (MSPyBS), Hospital Materno Infantil San Pablo (HMISP). Asunción, \\ Paraguay \\ ${ }^{3}$ Ministerio de Salud Pública y Bienestar Social (MSPyBS), Hospital Materno Infantil de Trinidad (HMIT). Asunción, \\ Paraguay \\ ${ }^{4}$ Ministerio de Salud Pública y Bienestar Social (MSPyBS), Centro de Salud N¹0 (CS N¹0). Asunción, Paraguay \\ ${ }^{5}$ Instituto de Enfermedades Infecciosas (INEI) - ANLIS "Dr. Carlos G. Malbrán", Servicio de Virus Oncogénicos, \\ Laboratorio Nacional y Regional de Referencia de VPH. Buenos Aires, Argentina
}

Cómo referenciar este artículo/

How to reference this article:
Bobadilla $M L$, Villagra $\mathrm{V}$, Zorrilla $M E$, Olmedo $G$, Riveros MC, Franco F, et al. Detección y tipificación del Virus Papiloma Humano en el marco del tamizaje virológico para la detección de lesiones del cuello uterino en Asunción, Paraguay. Mem. Inst. Investig. Cienc. Salud. 2019; 17(1): 6-15

\section{R E S U M E N}

En Paraguay la incidencia de cáncer de cuello uterino (CCU) es superior a las observadas en otros países de la región. El agente etiológico asociado al CCU es el virus papiloma humano (VPH), esencialmente tipos de alto riesgo oncogénicos. El objetivo es describir aspectos epidemiológicos de la infección genital por el virus papiloma humano de alto riesgo (VPHAR) en mujeres de 25 a 64 años que consultaron en servicios de Patología Cervical del MSPyBS, de mayo a diciembre de 2013. Se utilizó el Cobas 4800 HPV Test (Roche) que permite la detección individual de VPH-16 y VPH-18 y un pool de otros VPH-AR que incluye 12 genotipos de alto riesgo. Los otros VPH-AR fueron tipificados por hibridación reversa en línea (RLB). Entre las 495 mujeres incluidas, se detectaron 72 casos positivos $(14,5 \%)$ de VPH-AR. Se identificaron 19 tipos virales; siendo el más frecuente VPH-16 (2,1\%), seguido del VPH-31, 33, 58 y 66; el VPH-18 aparece en sexto lugar. Este trabajo aporta los primeros datos sobre la implementación de técnicas moleculares para detección y tipificación de VPH como parte del sistema de salud pública de Paraguay. El predominio de VPH-16, confirma su amplia circulación a nivel mundial y dado su mayor potencial oncogénico, representa una alerta a considerar, en especial en las mujeres mayores de 30 años portadoras de una infección persistente. Estos resultados apoyan la importancia de la implementación criteriosa y la utilización apropiada de las pruebas moleculares actualmente disponibles para la prevención y control del CCU.

Palabras clave: virus papiloma humano, Cobas 4800 HPV-Test, genotipificación, cáncer de cuello uterino.

\section{Detection and typing of Human Papilloma Virus during a virological screening for detection of cervical lesions in Asunción, Paraguay}

\section{A B S T R A C T}

In Paraguay the incidence of cervical cancer (CC) is higher than those observed in other countries of the region. The etiological agent associated with $\mathrm{CC}$ is human papilloma virus (HPV), essentially oncogenic high-risk types. The objective was to describe epidemiological 
aspects of genital infection by high-risk human papilloma virus (HR-HPV) in women aged 25 to 64 years who consulted in services of Cervical Pathology of the MSPyBS, from May to December 2013. We used the Cobas 4800 HPV Test (Roche) that allows the individual detection of HPV-16 and HPV-18 and a pool of other HR-HPV including 12 high-risk genotypes. Other HR-HPV were typed by reverse line hybridization (RLB). Among the 495 women included in the study, 72 positive cases $(14.5 \%)$ of HR-HPV were detected. We identified 19 viral types; the most frequent was HPV-16 (2.1\%), followed by HPV-31, 33, 58 and 66; HPV-18 appears in sixth place. This work provides the first data on the implementation of molecular techniques for the detection and typing of HPV as part of the public health system in Paraguay. The predominance of HPV-16 confirms its wide circulation worldwide and, given its greater oncogenic potential, represents an alert to be considered, especially in women over 30 years of age who carry a persistent infection. These data support the importance of the sensible implementation and the appropriate use of the molecular tests currently available for the prevention and control of the CC.

Keywords: human papilloma virus, Cobas 4800 HPV-Test, genotyping, cervical cancer.

\section{INTRODUCCIÓN}

El cáncer de cuello uterino (CCU) es el cuarto tipo de cáncer más común que afecta a las mujeres en el mundo y la primera causa de muerte por cáncer en mujeres en países en vías de desarrollo. A nivel mundial se estiman aproximadamente 570.000 casos nuevos y 311.000 muertes al año. La tasa de incidencia estandarizada por edad en América del Sur es 15,2 por 100.000 mujeres y en Paraguay la incidencia es muy superior a las observadas en otros países de la región, siendo la tasa de incidencia 31,5 por 100.000 mujeres y la tasa de mortalidad es de 16,0 por $100.000^{(1)}$.

El agente etiológico asociado al CCU es el virus papiloma humano (VPH), esencialmente los tipos de alto riesgo oncogénicos. La infección genital por el VPH constituye una de las infecciones de transmisión sexual (ITS) más frecuentes, se estima que hasta un $80 \%$ de las mujeres padecen la infección activa por VPH en algún momento de su vida ${ }^{(2)}$. La prevalencia de ADN de VPH en mujeres con citología normal a nivel mundial es aproximadamente del $10 \%$, encontrándose frecuencias más altas en África y América Latina $(20-30 \%)^{(3)}$.

Las lesiones intraepiteliales escamosas del cuello del útero constituyen las precursoras del CCU, por lo que su diagnóstico precoz es sumamente importante en la terapéutica y pronóstico de las pacientes; es por ello que, la detección de VPH representa actualmente un valioso componente de las guías clínicas para el tamizaje, manejo y tratamiento del CCU y sus lesiones precursoras.

Actualmente, el sistema público de salud dispone de la prueba de detección del ADN del $\mathrm{VPH}$, que ha sido introducida en una primera etapa como un proyecto piloto en tres centros de salud de la capital. El objetivo del presente trabajo es investigar los aspectos epidemiológicos de la infección genital por el virus papiloma humano de alto riesgo (VPHAR), a través del estudio de los virus circulantes en mujeres de 25 a 64 años que consultan en los servicios de Patología Cervical dependientes del Ministerio de Salud Pública y Bienestar Social de Paraguay, de mayo a diciembre de 2013.

\section{MATERIALES Y MÉTODOS \\ Población del estudio y recolección de muestras}

Estudio transversal en 495 mujeres atendidas en servicios de patología cervical de tres hospitales dependientes del sistema de salud pública de mayo a diciembre de 2013. Las pacientes acuden a estos servicios para el examen citológico cervicovaginal o Pap, método de tamizaje de rutina en el país. Aquellas pacientes que reunían criterios de selección preestablecidos (ser sexualmente activa, no estar embarazada, sin histerectomía total, y sin citología anormal preexistente o ausencia de controles anteriores en el contexto del tamizaje de rutina), fueron invitadas a someterse a la prueba del VPH. Las mismas firmaron un consentimiento informado y respondieron un cuestionario sobre características sociodemográficas y factores de riesgo asociados al CCU. El Comité de Ética del LCSP revisó y aprobó previamente el protocolo de estudio.

Durante la consulta, las pacientes fueron sometidas a un examen ginecológico completo y se recolectaron células cérvico-vaginales exfoliadas tanto para la detección del ADN de VPH-AR como para la preparación del frotis de Papanicolaou. 


\section{Detección y genotipificación de VPH-AR}

La detección de VPH-AR se realizó en el LCSP con el Cobas 4800 HPV Test (Roche Diagnostics, Mannheim, Alemania), ensayo cualitativo de PCR en tiempo real totalmente automatizado que permite la detección individual de los VPH-16 y VPH-18 y un pool de otros 12 genotipos $(31,33,35,39,45,51,52,56,58,5966$ y 68). Esta metodología utiliza la beta-globina como control interno de calidad para cada muestra. Los resultados fueron interpretados por el software del sistema como "negativo", VPH-16, VPH-18, Otros VPH-AR o la combinación de los tres últimos. La detección de VPH-AR se realizó sin conocer los resultados citológicos.

Las muestras positivas para Otros VPH-AR por el COBAS 4800 HPV Test fueron genotipificadas por hibridación reversa en línea (RLB) en el Servicio de Virus Oncogénicos del INEI-ANLIS "Dr. Carlos G. Malbrán" de Buenos Aires (Argentina). Brevemente, se realizó PCR genérica con cebadores PGMY09/11 biotinilados y los productos de PCR desnaturalizados se analizaron mediante RLB con un panel de sondas de VPH tipoespecíficas $^{(4)}$. Esta metodología permite la identificación de hasta 33 genotipos diferentes de VPH $(6,11,16,18,26,31,33,34,35,39,40,42,43,44,45,51,52,53,54,55,56,57 \mathrm{~b}$, $58,59 a, 59 b, 66,68,69,70,73,82,83$ y 84$)$.

\section{Citología}

El diagnóstico citológico fue realizado por cito-tecnólogos de cada servicio de Patología Cervical y los resultados fueron recolectados de los registros médicos de las pacientes incluidas en este estudio. Los mismos fueron clasificados según el sistema Bethesda $2001^{(5)}$.

\section{Análisis estadístico}

Se emplearon herramientas de estadística descriptiva para el análisis de las características epidemiológicas de los pacientes, calculando los promedios y las desviaciones estándar para las variables cuantitativas, frecuencias y porcentajes para las cualitativas. Para el estudio de la relación entre variables cualitativas se utilizó la prueba de chicuadrado. La significancia estadística se asumió con un valor de $\mathrm{p}$ igual o menor que 0,05. Los análisis se realizaron con Epi-Info 2002 (Centros para el Control y la Prevención de Enfermedades, Atlanta, GA, EEUU).

\section{RESULTADOS}

El estudio fue realizado en 495 mujeres atendidas en servicios de patología cervical de tres hospitales dependientes del sistema de salud pública de mayo a diciembre de 2013. Fueron incluidas mujeres de 25 a 64 años, con una edad media de $39 \pm 10$ años. Se encontraron 72 casos positivos de VPH-AR con una prevalencia global de $14,5 \%$ (IC $95 \%$ : $11,6$ a 18,0$)$.

Las características socio-demográficas, antecedentes ginecológicos y factores de riesgos asociados a la infección por el VPH que fueron investigadas se presentan en la Tabla 1. Puede observarse asociaciones significativas entre la infección por VPH-AR y la edad de la paciente (OR 2,69 - IC $95 \%$ : 1,53 - 4,73), el lugar de residencia (OR 2,10 - IC $95 \%$ : 1,23 3,61 ), el uso de anticonceptivos hormonales (OR 1,69- IC $95 \%$ : 0,97 - 2,94) y el antecedente de otras infecciones del tracto genital femenino (OR 1,79- IC $95 \%: 1,04-3,05$ ). 
Tabla 1. Características socio-demográficas y antecedentes ginecológicos de las pacientes estudiadas. $(n=495)$

\begin{tabular}{|c|c|c|c|}
\hline Características & $\begin{array}{l}\text { Positivos para VPH-AR } \\
\text { n }(\% \text { IC } 95 \%)\end{array}$ & $\boldsymbol{p}$ & OR (IC $95 \%)$ \\
\hline \multicolumn{4}{|l|}{ Edad (años) } \\
\hline Menos de 30 & $28\left(26\right.$ IC $\left._{95 \%} 18-35\right)$ & \multirow{2}{*}{0,0002} & \multirow{2}{*}{$2,69(2,53-4,73)$} \\
\hline 30 o más & $44\left(11\right.$ IC $\left._{95 \%} 9-15\right)$ & & \\
\hline \multicolumn{4}{|l|}{ Lugar de residencia } \\
\hline Asunción & $43\left(20 \mathrm{IC}_{95 \%} 15-26\right)$ & \multirow{2}{*}{0,004} & \multirow{2}{*}{$2,10(1,23-3,61)$} \\
\hline Otras localidades & $29\left(11 \mathrm{IC}_{95 \%} 7-15\right)$ & & \\
\hline \multicolumn{4}{|l|}{ Nivel de Educación } \\
\hline Primaria & $25\left(15\right.$ IC $\left._{95 \%} 10-22\right)$ & \multirow{2}{*}{0,76} & \multirow{2}{*}{$0,92(0,54-1,56)$} \\
\hline Secundaria o más & $47\left(14 \mathrm{IC}_{95 \%} 11-19\right)$ & & \\
\hline \multicolumn{4}{|l|}{ Ocupación } \\
\hline Ama de casa & $53(14$ IC95\% $11-18)$ & \multirow{2}{*}{0,68} & \multirow{2}{*}{$1,13(0,64-1,99)$} \\
\hline Empleo formal & $19(16$ IC95\% $10-23)$ & & \\
\hline \multicolumn{4}{|c|}{$\begin{array}{l}\text { Edad de la primera } \\
\text { relación sexual (años) }\end{array}$} \\
\hline 18 o menos & $40\left(13\right.$ IC $\left._{95 \%} 10-18\right)$ & \multirow{2}{*}{0,32} & \multirow{2}{*}{$1,29(0,78-2,13)$} \\
\hline Más de 18 & $32\left(17 \mathrm{IC}_{95 \%} 12-23\right)$ & & \\
\hline \multicolumn{4}{|c|}{ Nro. de parejas sexuales } \\
\hline $\begin{array}{l}1 \text { ó } 2 \\
3 \text { o más }\end{array}$ & $\begin{array}{l}58\left(14 \mathrm{IC}_{95 \%} 11-18\right) \\
14\left(17 \mathrm{I} \mathrm{C}_{95 \%} 10-27\right)\end{array}$ & 0,51 & $1,24(0,65-2,34)$ \\
\hline \multicolumn{4}{|c|}{ Vive con compañero sexual } \\
\hline $\begin{array}{l}\text { SI } \\
\text { NO }\end{array}$ & $\begin{array}{l}37\left(12 \text { IC }_{95 \%} 9-17\right) \\
35(17 \text { IC } 95 \% 12-23)\end{array}$ & 0,24 & $0,74(0,45-1,22)$ \\
\hline \multicolumn{4}{|l|}{ Paridad } \\
\hline 1 a 3 & $45\left(16\right.$ IC $\left._{95 \%} 12-20\right)$ & \multirow{2}{*}{0,10} & \multirow{2}{*}{$0,56(0,28-1,13)$} \\
\hline 4 ó más & $11\left(9 \mathrm{IC}_{95 \%} 5-16\right)$ & & \\
\hline \multicolumn{4}{|c|}{ Uso de anticonceptivos hormonales } \\
\hline SI & $27\left(19\right.$ IC $\left._{95 \%} 13-27\right)$ & \multirow{2}{*}{0,049} & \multirow{2}{*}{$1,69(0,97-2,94)$} \\
\hline NO & $45\left(13\right.$ IC $\left._{95 \%} 10-17\right)$ & & \\
\hline \multicolumn{4}{|c|}{$\begin{array}{l}\text { Antecedentes de otras infecciones } \\
\text { del tracto genital femenino }\end{array}$} \\
\hline SI & $33\left(19\right.$ IC $\left._{95 \%} 14-26\right)$ & 0024 & \\
\hline NO & $39\left(12\right.$ IC $\left._{95 \%} 9-16\right)$ & 0,024 & $1,19(1,08-2,90)$ \\
\hline Pap previo & & & \\
\hline SI & $62\left(14 \mathrm{IC}_{95 \%} 11-18\right)$ & & \\
\hline NO & $10\left(17\right.$ IC $\left._{95 \%} 9-29\right)$ & 0,54 & $0,19(0,38-1,65)$ \\
\hline Ultimo Pap & & & \\
\hline 1 año o menos & $41\left(16\right.$ IC $\left._{95 \%} 11-21\right)$ & 0.28 & $0.74(0.42-1.30)$ \\
\hline Más de 1 año & $21\left(12 \mathrm{IC}_{95 \%} 8-18\right)$ & 0,20 & $0,14(0,42-1,30)$ \\
\hline Hábito de fumar & & & \\
\hline SI & $8\left(23 \mathrm{IC}_{95 \%} 10-40\right)$ & 0.15 & $183(080-421)$ \\
\hline NO & $64\left(14 \mathrm{IC}_{95 \%} 11-17\right)$ & 0,15 & $1,83(0,80-4,21)$ \\
\hline
\end{tabular}

El mayor número de casos se registró en el grupo de mujeres menores de 30 años, siendo la edad promedio de 35,2 \pm 9,0 años. Como se puede ver en la Tabla 2, la infección por VPH-AR disminuye con la edad, siendo menos frecuente en mujeres de 50 años o más. EI VPH-16 se encontró en casi todos los grupos de edad, a diferencia del VPH-18 que se observó solo en mujeres menores de 40 años. Los Otros VPH-AR se distribuyen constantemente en todos los grupos de edad. 
Tabla 2. Distribución de VPH-16, VPH-18 y Otros VPH-AR por grupo etario.

\begin{tabular}{|c|c|c|c|c|c|}
\hline $\begin{array}{l}\text { Edad } \\
\text { (años) }\end{array}$ & $\begin{array}{l}\text { No de } \\
\text { Muestras }\end{array}$ & $\begin{array}{l}\text { VPH-16 } \\
\text { n }\left(\% ; \text { IC }_{95 \%}\right) *\end{array}$ & $\begin{array}{l}\text { VPH-18 } \\
\text { n }\left(\% ; \text { IC }_{95 \%}\right) *\end{array}$ & $\begin{array}{l}\text { OTROS VPH-AR } \\
\text { n }\left(\% ; \mathbf{I C}_{95 \%}\right) * *\end{array}$ & $\begin{array}{l}\text { Total de VPH-AR } \\
\text { n }\left(\% ; \text { IC }_{95 \%}\right)\end{array}$ \\
\hline Media \pm DE & - & $35,2 \pm 9,0$ & $29,3 \pm 3,8$ & $35,2 \pm 9,9$ & $34,9 \pm 9,5$ \\
\hline$<30$ & 109 & $7(6,4 ; 2,6-12,8)$ & $2(1,8 ; 0,2-6,5)$ & $19(17,4 ; 10,8-25,9)$ & $28(25,7 ; 17,8-34,9)$ \\
\hline 30 a 39 & 168 & $6(3,6 ; 1,3-7,6)$ & $2(1,2 ; 0,1-4,2)$ & $16(9,5 ; 5,5-15,0)$ & $24(14,3 ; 9,4-20,4)$ \\
\hline 40 a 49 & 133 & $3(2,3 ; 0,5-6,5)$ & - & $12(9,0 ; 4,7-15,2)$ & $15(11,3 ; 6,5-17,9)$ \\
\hline$\geq \mathbf{5 0}$ & 85 & $1(1,2 ; 0,0-6,4)$ & - & $4(4,7 ; 1,3-11,6)$ & $5(5,9 ; 1,9-13,2)$ \\
\hline Total & 495 & $\begin{array}{l}13(18,1 ; 9,1- \\
27,0)\end{array}$ & $2(2,8 ; 1,0-6,6)$ & $13(18,1 ; 9,1-27,0)$ & $\begin{array}{l}72(14,5 ; 11,6- \\
18,0)\end{array}$ \\
\hline
\end{tabular}

(*) Infecciones únicas o múltiples;

(**) Otros VPH-AR $(31,33,35,39,45,51,52,56,58,59,66$ y 68), excluyendo co-infecciones con VPH-16 o VPH18.

Con respecto a los datos citológicos, el 93,1\% (461/495) de las muestras incluidas en el estudio no tenían lesión intraepitelial escamosa (N-SIL). La Tabla 3 muestra los resultados de la detección de VPH-AR según la gravedad de la lesión. Se detectó VPH-AR en 11,9\% de los pacientes con N-SIL y la frecuencia de detección de VPH-AR aumentó en las pacientes con anormalidades citológicas de mayor grado.

Tabla 3. Distribución de VPH-16, VPH-18 y Otros VPH-AR según diagnóstico citológico.

\begin{tabular}{|c|c|c|c|c|c|}
\hline \multirow{2}{*}{ Resultado } & \multicolumn{4}{|c|}{ Citología } & \multirow{2}{*}{ - Total } \\
\hline & N-SIL & ASC-US & L-SIL & H-SIL & \\
\hline $\begin{array}{l}\text { Negativos para } \\
\text { VPH-AR ( } \% \\
\text { IC } 9 \% \text { ) }\end{array}$ & $\begin{array}{l}406(88,1 ; 84,7- \\
90,8)\end{array}$ & $\begin{array}{l}2(50,0 ; 15,0- \\
85,0)\end{array}$ & $\begin{array}{l}14(56,0 ; 34,9- \\
75,6)\end{array}$ & $\begin{array}{l}1(20,0 ; 0,5- \\
71,6)\end{array}$ & $\begin{array}{l}423(85,5 ; 15,0 \\
85,0)\end{array}$ \\
\hline $\begin{array}{l}\text { Positivos para } \\
\text { VPH-AR ( \% } \\
\text { IC } 95 \%)\end{array}$ & $\begin{array}{l}55(11,9 ; 9,2- \\
15,3)\end{array}$ & $\begin{array}{l}2(50,0 ; 15,0- \\
85,0)\end{array}$ & $\begin{array}{l}11(44,0 ; 24,4- \\
65,1)\end{array}$ & $\begin{array}{l}4(80,0 ; 28,4- \\
99,5)\end{array}$ & $\begin{array}{l}72(14,5 ; 11,6- \\
18,0)\end{array}$ \\
\hline VPH-16 & $10(2,2 ; 1,2-3,9)$ & $\begin{array}{l}1(25,0 ; 4,6- \\
69,9)\end{array}$ & $\begin{array}{l}4(16,0 ; 6,4- \\
34,7)\end{array}$ & $\begin{array}{l}2(40,0 ; 11,8- \\
76,9)\end{array}$ & $\begin{array}{l}17(3,4 ; 2,2- \\
5,5)\end{array}$ \\
\hline VPH-18 & $2(0,4 ; 0,1-1,6)$ & - & $\begin{array}{l}2(8,0 ; 2,2- \\
25,0)\end{array}$ & - & $4(0,8 ; 0,3-2,1)$ \\
\hline Otros VPH-AR & $\begin{array}{l}43(9,3 ; 7,0- \\
12,3)\end{array}$ & $\begin{array}{l}1(25,0 ; 4,6- \\
69,9)\end{array}$ & $\begin{array}{l}5(20,0 ; 8,9- \\
39,1)\end{array}$ & $\begin{array}{l}2(40,0 ; 11,8- \\
76,9)\end{array}$ & $\begin{array}{l}51(10,3 ; 7,9- \\
13,3)\end{array}$ \\
\hline Total de muestras & 461 & 4 & 25 & 5 & 495 \\
\hline
\end{tabular}

N-SIL: negativo para lesión escamosa intraepitelial; ASC-US: presencia de células escamosas alteradas de significado incierto; L-SIL: lesión escamosa intraepitelial de bajo grado; H-SIL: lesión escamosa intraepitelial de alto grado.

Fueron estudiadas por PCR-RLB las muestras positivas para Otros VPH-AR por el Cobas 4800 HPV Test, incluyendo las co-infecciones con VPH-16 o VPH-18. En total 19 tipos virales distintos pudieron identificarse once de alto riesgo $(16,18,31,33,39,45,51,52,56,58,59)$; tres de probable alto riesgo $(26,66,68)$ y cinco de bajo riesgo $(6,43,53,55,70)$. No pudo determinarse el genotipo en diez muestras. El genotipo más frecuente fue el VPH-16 $(2,1 \%)$, seguido del VPH-31, 33, 58 y 66 . El VPH-18 aparece en sexto lugar.

Se encontró una frecuencia similar de infecciones múltiples en las mujeres con citología negativa $(29,3 \%$, IC $95 \% 16,1-45,5)$ y en las mujeres con ASC-US o más $\left(26,7 \% ;\right.$ IC $_{95 \%} 7,8-$ $55,1)(p=0,84)$. En la Figura 1 se muestra la distribución de frecuencia de los diferentes genotipos en mujeres con N-SIL y en mujeres con ASC-US o más. 
Figura 1. Distribución de genotipos virales en N-SIL y ASC-US o más.

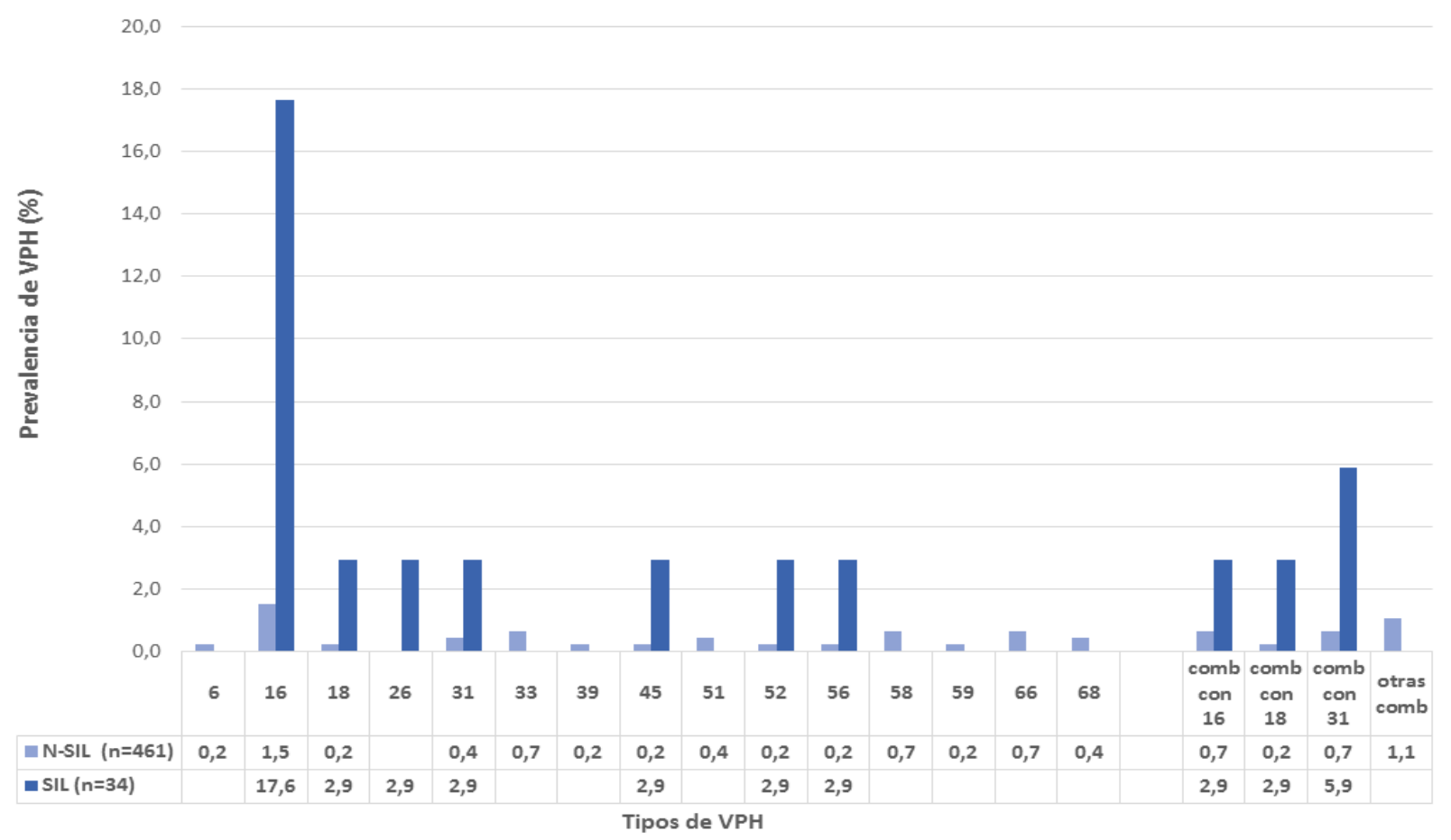

\section{DISCUSIÓN}

Este trabajo muestra los primeros resultados obtenidos con la implementación de técnicas moleculares para detección y tipificación de VPH como parte del sistema de salud pública de Paraguay, llevado a cabo como un estudio piloto dentro de los programas nacionales de tamizaje, realizado en un contexto asistencial en consultorios ginecológicos.

Fue detectado VPH-AR en el $11,9 \%$ de las pacientes con citología normal. Esto es bastante similar a lo descripto en un estudio nacional en mujeres paraguayas con citologías negativas para lesión escamosa intraepitelial que reporta una prevalencia del $21 \%$ para VPH genérico y del $12,1 \%$ para $\mathrm{VPH}-\mathrm{AR}^{(6)}$. Además, es semejante a lo observado en estudios poblacionales de la región, uno en Argentina con una prevalencia del $16,6 \%$ para VPH y del $12,1 \%$ para VPH-AR ${ }^{(7)}$; y otro en Chile que encontró una prevalencia del $23,2 \%$ para VPH y del $15,3 \%$ para $\mathrm{VPH}-\mathrm{AR}^{(8)}$. Además, es cercano a lo reportado en el meta-análisis en un millón de mujeres con citología normal, de los cinco continentes, donde la prevalencia estandarizada de VPH para Sudamérica fue del 15,3\%. Cabe destacar que, en ese estudio, la prevalencia estandarizada de VPH por países mostró un amplio rango de variabilidad (del $1,6 \%$ al $41,96 \%)^{(9)}$. Esto indicaría que la frecuencia de infección es afectada por las características étnicas, culturales y de desarrollo, propias, lo que justificaría a su vez la semejanza de nuestros datos con aquéllos obtenidos en otros países de Latinoamérica.

Así también, el estudio ATHENA, diseñado para la evaluación clínica del Cobas $4800 \mathrm{HPV}$ Test, encontró VPH-AR en el 6,7\% de 32.260 mujeres con citología normal, de 30 años o más, en los EEUU ${ }^{(10)}$, valor considerablemente menor a lo reportado en este trabajo. Se ha demostrado que la prevalencia de VPH en países más desarrollados es sustancialmente más baja $(6,2 \%)^{(9)}$. Otro factor que podría contribuir a esta diferencia es el rango de edad de la población, que en este estudio incluyó algunas mujeres menores de 30 años. Es bien conocido que por debajo de 30 años hay un marcado pico de prevalencia de VPH debido a la proximidad del debut sexual, fuertemente relacionado a la primo infección ${ }^{(11)}$.

Está bien establecido que la infección cervical por VPH es edad dependiente ${ }^{(12)}$, mostrando un patrón general que disminuye con la edad. En este trabajo, se observó una disminución de la prevalencia de VPH-AR en función de la edad, sin un aumento en el grupo de mujeres de 45-50 años como lo reportado para Sudamérica por Bruni $L$ et al. ${ }^{(9)}$, sin embargo, coincide con estudios poblacionales de Argentina y Chile ${ }^{(7,8)}$. La disminución progresiva de la presencia de VPH-AR en mujeres mayores de 30 años se explica por el carácter auto-limitado y el control de la infección por la acción del sistema inmune del 
hospedador (infecciones transitorias), lo que hace que el virus se haga indetectable ${ }^{(13)}$. En algunos casos, la respuesta inmune falla y lleva a una infección persistente, mayormente por tipos de VPH-AR; esta situación de persistencia viral aumenta la posibilidad de progresión a una lesión preneoplásica grave, que en caso de no ser tratada oportunamente podría alcanzar el estadio de carcinoma invasor ${ }^{(13-15)}$.

El efecto del tamizaje sobre la frecuencia viral ha sido descripto y se considera una de las razones por las cuales las curvas de prevalencia para América Central y Sudamérica muestran valores mayores que las de América del Norte ${ }^{(16)}$. Esto podría justificar en parte, la disminución de frecuencia de VPH-AR con edad observada en este trabajo, pues las mujeres estudiadas corresponden mayormente a una población frecuentemente controlada, reclutadas al acudir a los servicios de salud para la realización del estudio citológico de rutina; el $88 \%$ de ellas se había realizado una prueba de Papanicolaou anteriormente y el $60 \%$ lo había hecho en el último año.

En el $29,3 \%$ ( IC $\left._{95 \%} 16,1-45,5\right)$ de las pacientes con citología negativa, se identificaron infecciones por más de un tipo viral; frecuencia ligeramente menor al $20 \%$ reportado por Bruni et al. ${ }^{(9)}$, pero muy similar al $30,1 \%$ reportado en una población de mujeres paraguayas $^{(17)}$. En la actualidad aún no está completamente aclarado el papel de las infecciones múltiples en el desarrollo de lesiones cervicales. En algunos trabajos, han sido asociadas con un riesgo mayor de neoplasia cervical intraepitelial ${ }^{(18,19)}$; mientras que, en otros, en coincidencia con nuestros hallazgos, no se ha observado diferencia significativa en la contribución para el desarrollo de neoplasia cervical intraepitelial y CCU en mujeres con infecciones simples o múltiples ${ }^{(20,21)}$. Sin embargo, hay evidencia de que, más allá de la presencia de múltiples tipos virales en una muestra, sólo uno de los VPH-AR es el que va a conducir la potencial transformación maligna ${ }^{(22)}$.

El VPH-16 es el virus más frecuente independientemente del diagnóstico citológico (9; 16 ; 23-24). En coincidencia con esto, se observó en este estudio que el VPH-16 es el tipo más frecuente $(2,1 \%)$ en pacientes con citología normal, similar a lo observado en Paraguay, $(4,3 \%)^{(6)}$; en la región de Latinoamérica y el Caribe $(3,3 \%)$ y a nivel mundial $(3,2 \%)^{(9,16)}$. Otros tipos comúnmente detectados fueron VPH-31, 33 y 58 y el 66 . El VHP-18 aparece en sexto lugar. A nivel mundial, el $\mathrm{VPH}-18(1,4 \%)$ se ubica en segundo lugar después VPH-16 en citología normal. Sin embargo, al analizar su distribución se observa menor frecuencia en diferentes regiones ${ }^{(3,9,16)}$.

Numerosos autores han señalado que las mujeres con infecciones persistentes por VPH16 poseen una mayor probabilidad de desarrollar lesiones precancerosas al ser comparadas con mujeres infectadas por otros tipos virales. Kjaer et al. observaron que mujeres con citología normal e infección persistente por $\mathrm{VPH}-16$ poseen aproximadamente un $30 \%$ de probabilidad de desarrollar lesiones de alto grado en un período de 12 meses $^{(15,25-27)}$. Esto da relevancia al presente estudio, ya que la mayoría de las mujeres que se identificaron como portadoras de infecciones por VPH-AR presentaban citología normal; sin embargo, la presencia viral, en especial en las mayores de 30 años, las ubica en un grupo de mayor riesgo de una potencial persistencia viral, instancia previa al probable desarrollo de lesiones graves.

El hecho de que la mayoría de las pacientes infectadas, aún con VPH-AR no progresen a $\mathrm{CCU}$, indica que la presencia viral, si bien es necesaria, no es suficiente para general la neoplasia; otros factores contribuirían para la transformación maligna. Se han considerado entre ellos a la multiparidad, las coinfecciones con otras ITS, factores del hospedador vinculados con la respuesta inmune y la acción hormonal, el tabaquismo, estado nutricional, entre otros ${ }^{(21,28-31)}$.

Se observó una marcada disminución en la infección por VPH con la edad. Estos resultados son consistentes con investigaciones anteriores que han demostrado que la prevalencia del VPH en mujeres con citología normal disminuye con la edad, siendo las mujeres $<25$ años las que tienen tasas sustancialmente más altas ${ }^{(16,32-33)}$. Se ha sugerido que las células cervicales son más susceptibles a la infección por VPH a edades tempranas ${ }^{(34)}$. Además, entre las mujeres más jóvenes, la mayor actividad sexual y la menor probabilidad de parejas estables, constituyen un fuerte predictor de la infección.

En Asunción la prevalencia de VPH fue significativamente mayor que en las otras localidades $(p=0,004)$. Si bien no se observó una marcada diferencia entre el número de parejas sexuales y la edad del inicio de las relaciones sexuales entre mujeres de la capital y 
de localidades más pequeñas, se ha descripto en trabajos previos que las poblaciones urbanas y rurales muestran diferencias socioeconómicas y psicosociales (estilo y calidad de vida) que podrían explicar de alguna manera este resultado ${ }^{(35-36)}$.

La asociación entre el uso de anticonceptivos hormonales y la infección por VPH encontrada en el presente trabajo fue bastante débil $(p=0,049)$. Se ha propuesto que estrógenos o progestágenos aumentan la expresión de genes del VPH en el cuello uterino a través de receptores de progesterona y elementos de respuesta a hormonas en el genoma viral $^{(28)}$. Un meta-análisis halló que en las usuarias de anticonceptivos hormonales el riesgo de CCU aumenta con la duración de su uso y que dicho riesgo disminuye una vez que se interrumpe su utilización ${ }^{(37)}$. Sin embargo, aunque numerosos estudios proveen una fuerte evidencia de la relación causal del uso de anticonceptivos hormonales y el CCU, debe tenerse en cuenta que la mayoría de los datos disponibles corresponden al uso de anticonceptivos orales de primera generación, que difieren a los utilizados actualmente ${ }^{(38)}$.

Existe evidencia sobre el papel de otras infecciones del tracto genital femenino como cofactores para el desarrollo del CCU en mujeres VPH positivas. En la población estudiada se encontró una asociación estadísticamente significativa entre la infección por VPH-AR y el antecedente de estas infecciones $(p=0,024)$. Numerosos trabajos han considerado que coinfecciones con el virus Herpes simple tipo 2 (HVS-2) o Chlamydia trachomatis (CT) predisponen al desarrollo del CCU en mujeres con infecciones persistentes de VPH-AR. Se ha demostrado que las infecciones por HVS-2 y CT generan una respuesta inflamatoria, asociada con la liberación de radicales libres que producen inestabilidad genética ${ }^{(39)}$. Otros microorganismos, como Gardnerella sp, Candida sp, Trichomonas sp, Mycoplasma hominis, Ureaplasma urealyticum y Treponema pallidum han sido asociados con procesos inflamatorios en el cuello uterino, hecho que podría facilitar la entrada del $\mathrm{VPH}^{(40-41)}$ y desfavorecer la respuesta inmune.

Este estudio aporta los primeros datos sobre la implementación de técnicas moleculares para detección y tipificación de VPH como parte del sistema de salud pública de Paraguay. La alta frecuencia de infección por VPH-AR encontrada en pacientes con citología normal, similar a la hallada en otros países de la región, representa información de valor a la hora de decidir políticas sanitarias de prevención de lesiones precancerosas y CCU. Este hallazgo explicaría en parte, la elevada incidencia de esta neoplasia en Paraguay. Los tipos viales hallados coinciden con los informados en otras regiones del mundo. El tipo más frecuente fue el VPH-16; seguido, en orden decreciente, por el VPH-31, 39, 58 y 33 . El predominio de VPH-16, confirma la amplia circulación de este virus a nivel mundial y dado su mayor potencial oncogénico, señala una alerta a considerar, en especial en las mujeres por encima de los 30 años portadoras de una infección persistente. Estos datos apoyan la importancia de la implementación criteriosa y la utilización apropiada de las pruebas moleculares actualmente disponibles para la prevención y control del CCU. Su uso en el contexto de programas nacionales como tamizaje primario podría significar una herramienta de gran valor y ayuda para la óptima selección del tratamiento y seguimiento de las mujeres. Esto brinda un escenario sumamente promisorio en cuanto a las posibilidades de mejorar el manejo clínico de las pacientes con patología cervical y la prevención del CCU.

\section{REFERENCIAS BIBLIOGRAFICAS}

1. Global Cancer Observatory. International Agency for Research on Cancer. WHO 2018. Estimated age-standarized cancer incidence, mortality and prevalence worldwide in 2018. Disponible en http://gco.iarc.fr/

2. Bosch FX, Burchell AN, Schiffman $M$, Giuliano AR, de Sanjosé S, Bruni $L$ et al. Epidemiology and natural history of human papillomavirus infections and type specific implications in cervical neoplasia. Vaccine. 2008; 26S:K1-K16.

3. Clifford GM, Gallius S, Herrero R, Muñoz N, Snijders PJF, Vaccarella $S$ et al. Worldwide distribution of human papillomavirus types in cytologically normal women in the International Agency for Research on Cancer
HPV prevalence surveys: a pooled analysis. Lancet. 2005;366: 991-98.

4. Estrade C, Menoud PA, Nardelli-Haefliger D, Sahli R. Validation of a low-cost human papillomavirus genotyping assay based on PGMY PCR and reverse blotting hybridization with reusable membranes. J Clin Microbiol. 2011; 49 (10): 3474-81.

5. Solomon D, Davey D, Kurman R, Moriaty A, $O^{\prime}$ Connor $D$, Prey $M$ et al. Terminology for reporting results of cervical cytology. JAMA. 2002; 267(16):2114-19.

6. Mendoza LP, Arbiza J, Páez M, Kasamatsu E, Castro A, Giménez $G$ et al. Características clínico-demográficas y tipificación del virus de papiloma humano en mujeres paraguayas 
con citologías negativas para lesión escamosa intraepitelial. Mem. Inst. Investig. Cienc. Salud. 2012; Vol. 10(1):46-55

7. Matos E, Loria D, Amestoy GM, Herrera L, Prince MA, Moreno $\mathrm{J}$ et al. Prevalence of Human Papillomavirus Infection Among Women in Concordia, Argentina: A Population-Based Study. Sexually transmitted diseases. 2003;30(8):593-99.

8. Ferreccio $C$, Prado $R$, Luzoro A. Prevalencia poblacional y distribución por edad del virus papiloma humano entre mujeres en Santiago, Chile. Boletín Escuela de Medicina. 2005; 30 (1): 34-9.

9. Bruni L, Diaz M, Castellsagué $M$, Ferrer $E$, Bosch FX and de Sanjosé S. Cervical human papillomavirus prevalence in 5 continents: meta-analysis of 1 million women with normal cytological findings. J Infect Dis. 2010 Dec 15;202(12):1789-99.

10. Wright TC, Stoler MH, Sharma A, Zhang G, Behrens C , Wright TL. Evaluation of HPV-16 and HPV-18 genotyping for the triage of women with high-risk HPV+ cytologynegative results. Am J Clin Pathol. 2011 Oct; $136(4): 578-86$.

11. Wellings $K$, Collumbien $M$, Slaymaker $E$ Singh S, Hodges $Z$, Patel $D$, et al. Sexual behavior in context: a global perspective. Lancet. 2006;368:1706-28.

12. Schiffman $M$, Castle $P E$, Jeronimo J, Rodriguez AC, Wacholder S. Human papillomavirus and cervical cancer. The Lancet. 2007;370(9590):890-907.

13. Stanley MA, Sterling JC. Host responses to infection with human papillomavirus. In Human Papillomavirus. Karger Publishers. 2014; 45:58-74.

14. Rodríguez AC, Schiffman M, Herrero R et al. Rapid clearance of human papillomavirus and implications for clinical focus on persistent infections. J Natl Cancer Inst. 2008;100;513-17.

15. Castle PE, Solomeno D, Schiffman M, Wheeler CM. Human papillomavirus type 16 infections and 2-year absolute risk of cervical precancer in women with equivocal or mild cytologic abnormalities. J Natl Cancer Inst 2005; 97: 1066-71.

16. De Sanjosé $S$, Diaz $M$, Castellsagué $X$, Clifford G, Bruni L, Muñoz N and Bosch FX. Worldwide prevalence and genotype distribution of cervical human papillomavirus DNA in women with normal cytology: a meta-analysis. The Lancet infectious diseases.2007;7(7): 453-9.

17. Cardozo Segovia FM, Mendoza Torres LP, Páez Acchiardi GM, Mongelós Dacunte PE, Castro Matto AM, Giménez Ortigoza G. Detección molecular de infecciones múltiples en mujeres con y sin lesión de cuello uterino positivas para el virus del papiloma humano de alto riesgo, período 2007-2011. Mem.
Inst. Investig. Cienc. Salud. 2012 Dec; 10(2):05-12.

18. Sasagawa $T$, Basha $W$, Yamazaki $H$, Inoue M. High-risk and multiple human papillomavirus infections associated with cervical abnormalities in Japanese women. Cancer Epidemiol Biomark Prev. 2001; 10:45-52.

19. Van der Graaf $Y$, Molijn A, Doornewaard $H$, Quint W, Van Doorn LJ y Van den Tweel J. Human papillomavirus and the long-term risk of cervical neoplasia. Am. J. Epidemiol. 2002; 156:158-64.

20. Herrero R, Hildesheim A, Bratti C, Sherman ME et al. Population-based study of human papillomavirus infection and cervical neoplasia in rural Costa Rica. J Natl Cancer Inst. 2000;92:464-74.

21. Bosch FX, Lorincz A, Muñoz N, Meijer CJ y Shah KV. The causal relation between papillomavirus and cervical cancer. J Clin Pathol. 2002;55:244-65.

22. Quint W, Jenkins $D$, Molijn A, Struijk L, Van der Sandt M, Doobar J, Mols J, Van Hoof C, Hardt K, Struyf F, Colau B. One virus, one lesion - individual components of CIN lesions contain a specific HPV type. J Pathol. 2012; May227(1):62-71

23. Clifford GM, Smith JS, Aguado T, Franceschi S. Comparison of HPV type distribution in high-grade cervical lesions and cervical cancer: a meta-analysis. $\mathrm{Br} \mathrm{J}$ Cancer. 2003;89:101-5.

24. Clifford GM, Rana RK, Franceschi S, Smith JS, Gough G, Pimenta JM. Human papillomavirus genotype distribution in lowgrade cervical lesions: comparison by geographic region and with cervical cancer. Cancer Epidemiol Biomarkers Prev, 2005; 14: 1157-64.

25. Kjær SK, Frederiksen K, Munk C , Iftner T. Long-term absolute risk of cervical intraepithelial neoplasia grade 3 or worse following human papillomavirus infection: role of persistence. Journal of the National Cancer Institute. 2010;102(19):1478-88.

26. Schiffman $M$, Herrero $R$, Desalle $R$, Hildesheim A, Wacholder $S$, Rodriguez AC, Bratti MC, Sherman ME, Morales J, Guillén $D$, Alfaro $M$, Hutchinson $M$, Wright TC, Solomon D, Chen Z, Schussler J, Castle PE, Burk RD. The carcinogenicity of human papillomavirus types reflects viral evolution. Virology 2005, 337:76-84.

27. Khan MJ, Castle PE, Lorincz AT, Wacholder S, Sherman M, Scott DR, et al. The elevated 10 -year risk of cervical precancer and cancer in women with human papillomavirus (HPV) type 16 or 18 and the possible utility of type-specific HPV testing in clinical practice. J Nath Cancer Inst. 2005 Jul 20;97(14): 1072-79.

28. Muñoz N, Castellsagué $X$, de Gonzalez AB, Gissmann L. Chapter 1: HPV in the etiology 
of human cancer. Vaccine. 2006;24(suppl 3):S1-S10.

29. Anttila T, Saikku P, Koskela P, Bloigu A, Dillner J, Ikäheimo I et al. Serotypes of Chlamydia trachomatis and risk for development of cervical squamous cell carcinoma. JAMA. 2001;285(1):47-51.

30. Moreno V, Bosch FX, Muñoz N, Meijer CJ, Shah KV, Walboomers IM et al. Effect of oral contraceptives on risk of cervical cancer in women with human papillomavirus infection: The IARC multicentric case-control study. Lancet. 2002;359:1085-92.

31. Castellsague $X$, Muñoz N. Chapter 3: Cofactors in human papillomavirus carcinogenesis-role of parity, oral contraceptives, and tobacco smoking. J Natl Cancer Inst Monogr. 2003; 31:20-8.

32. Bosch FX, Burchell AN, Schiffman $M$, Giuliano AR, de Sanjosé $S$, Bruni $L$, Tortolero-Luna G, Krugar Kjaer S, Munoz N. Epidemiology and natural history of human papillomavirus infections and type specific implications in cervical neoplasia. Vaccine. 2008;26S:K1-K16.

33. Hibbitts S, Jones J, Powell N, Dallimore N, McRea J, Beer $H$, Tristram A, Fielder $H$, Fiander AN. Human papillomavirus prevalence in women attending routine cervical cancer screening in South Wales, UK: A cross-sectional study. $\mathrm{Br} \mathrm{J}$ Cancer. 2008;99:1929-33.

34. Muñoz N, Bosch FX, de Sanjosé S, Shah KV. The role of HPV in the etiology of cervical cancer. Mutation Research/Fundamental and Molecular Mechanisms of Mutagenesis. 1994;305(2):293-301.

35. Sharma K, Kathait A, Jain A, Kujur K, Raghuwanshi S, Bharti AC, et al. Higher prevalence of human papillomavirus infection in adolescent and young adult girls belonging to different Indian tribes with varied socio-sexual lifestyle. PlosS One. 2015 May 8;10(5):e0125693.

36. Vamos CA, Calvo AE, Daley EM, Giuliano AR y López Castillo H. Knowledge, Behavioral, and Sociocultural Factors Related to Human Papillomavirus Infection and Cervical Cancer Screening Among Inner-City Women in Panama. J Community Health. 2015 Dec;40(6):1047-56.
37. Beral V, Berrington de Gonzalez A, Colin D, Franceschi S, Goodhill A et al. International Collaboration of Epidemilogical Sudies of Cervical Cancer: Cervical cancer and hormonal contraceptives: collaborative reanalysis of individual data for 16.573 women with cervical cancer and 35.509 women without cervical cancer from 24 epidemiological studies. Lancet 2007; 370(9599):1609-21

38. Almonte M, Albero G, Molano M, Carcamo C, García PJ and Pérez G. Risk factors for human papillomavirus exposure and cofactors for cervical cancer in Latin America and the Caribbean. Vaccine. 2008;26:L16L36.

39. Castle PE, Jeronimo J, Schiffman M, Herrero $\mathrm{R}$, Rodríguez AC, Bratti MC et al. Age-related changes of the cervix influence human papillomavirus type distribution. Cancer Research. 2006;66(2):1218-24.

40. Roeters $A M$, Boon $M E$, van Haaften $M$, Vernooij F, Bontekoe $T$ and Heintz APM. Inflammatory events as detected in cervical smears and squamous intraepithelial lesions. Diagnostic cytopathology. 2010;38(2): 8593.

41. Oliveira FA, Pfleger V, Lang K, Heukelbach J, Miralles I, Fraga $F$ et al. Sexually transmitted infections, bacterial vaginosis, and candidiasis in women of reproductive age in rural Northeast Brazil: a population-based study. Memorias do Instituto Oswaldo Cruz. 2007;102(6):751-56. 